
\title{
R Resarach S Surare \\ Design and Manufacturing of Low Relative Humidity Chamber for Laser Processing of Lithium Metal
}

\section{Dongkyu Park}

Graduate School Kongju National University

Dongkyoung Lee ( $\nabla$ ldkkinka@kongju.ac.kr)

Graduate School Kongju National University

\section{Research Article}

Keywords: Manufacturing a chamber, Laser processing, Lithium metal, Gaussian beam

Posted Date: November 2nd, 2021

DOI: https://doi.org/10.21203/rs.3.rs-1029786/v1

License: (c) (i) This work is licensed under a Creative Commons Attribution 4.0 International License. Read Full License 


\title{
Design and Manufacturing of Low Relative Humidity Chamber for Laser Processing of Lithium Metal
}

\author{
Dongkyu Park ${ }^{1}$, Dongkyoung Lee Le, $^{1,2}$ † \\ ${ }^{\mathbf{1}}$ Department of Future Convergence Engineering, Graduate School Kongju National University, Cheonan, South \\ Korea \\ ${ }^{2}$ Department of Mechanical and Automotive Engineering, Kongju National University, Cheonan, South Korea \\ ${ }^{3}$ Center for Advanced Power Materials and Parts of Powder (CAMP ${ }^{2}$, Kongju National University, Cheonan \\ 31080, South Korea
}

${ }^{\dagger}$ Corresponding author: ldkkinka@kongju.ac.kr

\begin{abstract}
In order to overcome the energy density limitations of lithium-ion batteries consisting of graphite anodes, studies on lithium metal batteries (LMBs) have been actively conducted. However, most LMBs related studies focus on suppressing the growth of unpredictable dendrites. Research for production processes has been rarely conducted. In the paper, laser processing is introduced to improve the drawback of conventional processing for $\mathrm{Li}$ metal. Moreover, a low humidity maintenance chamber is manufactured to prevent oxidation of Li metal during laser processing because Li metal easily reacts with moisture. The chamber has a closed space that does not allow outside air to enter, and a glass that allows the laser beam to pass through is installed at the upper part. In addition, silica gel is installed to maintain low relative humidity. The dew point inside the chamber drops to $-17.4{ }^{\circ} \mathrm{C}$ in 1 hour. This result implies that the chamber can prevent Li metal oxidation. Next, we analyze the effect of transparent plate glass on laser beam with Gaussian distribution. Finally, it is confirmed through experiments that lithium metal is not contaminated by moisture during laser processing using a manufactured chamber.
\end{abstract}

Keywords: Manufacturing a chamber, Laser processing, Lithium metal, Gaussian beam 


\section{Introduction}

Over the past 30 years, the energy density, power density, and lifespan of lithium-ion batteries (LIBs) have been steadily developed and have good performance compared to lead-acid batteries, NiCd batteries, and Ni-MH batteries[1][2]. The development of LIBs has improved the performance of electric applications such as smartphones, laptops, tablets, and electric vehicles. Currently, the energy density of the commercialized LIBs is approximately $250 \mathrm{Wh} / \mathrm{kg}$, reaching almost theoretical capacity[3], [4]. Conventional LIBs have limited effective capacity enhancements due to the limitation of the theoretical capacity of graphite anodes $(370 \mathrm{mAh} / \mathrm{g})$ [5]. However, customers want to drive more distance by electric vehicles and use a wireless electronic device for a long time. For this reason, the development of new batteries that exceed the performance of LIBs consisting of graphite anode is actively underway.

Lithium (Li) metal is being actively discussed as an anode for the next generation batteries. Because the theoretical capacity of the Li metal has $3860 \mathrm{mAh} / \mathrm{g}$, which is about 10 times higher than that of graphite, and has the lowest reduction potential (3.04 V vs. the standard hydrogen electrode) and density $\left(0.534 \mathrm{~g} / \mathrm{cm}^{3}\right)$. Batteries consisting of Li metal anode are so-called Li metal batteries (LMBs) LMBs were developed before LIBs but were rarely used as secondary batteries [6]-[8]. LMBs have unpredictable Li dendrites growing on Li metal surface during operation. Li dendrites result in a reduction of lifespan, explosion, and low coulombic efficiency of batteries[9]. Currently, many studies are being conducted to suppress $\mathrm{Li}$ dendrite growth. $\mathrm{Li}$ et al.[10] produced a 3D porous $\mathrm{Cu}$ current collector/Li metal composite anode using copper mesh. The manufactured anode induced low local current density due to its large surface area and reduced interfacial resistance between electrolytes and electrodes. As a result, the formation of dendrites was suppressed and a stable solid electrolyte interphase layer was formed, so that the stability and lifespan of LMBs increased. Yang et al.[11] synthesized a mesoporous silica thin film (MSTF) on an anodic aluminum oxide (AAO) membrane through the polymer transfer method. When MSTF $\perp$ AAO separator was applied to Li-Li cells, It 
showed stable cycling performance at ultrahigh current density $\left(10 \mathrm{~mA} / \mathrm{cm}^{2}\right)$ by inducing a uniform $\mathrm{Li}^{+}$flux. Park et al.[12] produced patterns on Li metal surface through stamping techniques. During Li plating/stripping processes, $\mathrm{Li}^{+}$was induced and drained in fine patterns to suppress dendrite formation, enabling a stable cycle. However, mechanical processing methods such as stamping technique may easily contaminate tools when processing Li metal which has low Mohs hardness (0.6). Contaminated tools cause secondary contamination of the workpiece, which requires continuous cleaning. This can cause productivity to be reduced in the production process [13]. Therefore, a method to achieve high repeatability, fast production speed, and high quality in Li metal processing is required. Laser processing has the advantages of contactless processing, high precision, fast process speed and easy automation, and has already successfully replaced conventional process methods in various fields[14]-[19]. However, few studies have been conducted on the interaction characteristic between laser and Li metal to apply laser processing to Li metal. This is because, in order to handle Li metal, a very low relative humidity (RH) environment such as a dry room or glove box should be required. Figure 1 shows the Li metal exposed to air at a temperature of $25^{\circ} \mathrm{C}$ and $\mathrm{RH}$ of $40.0 \%$ over time. Silver-white surface can be observed on initial Li metal, and a black coating layer is gradually formed on Li metal over time. According to the literature review, this black coated layer is formed by the reaction of Li metal with $\mathrm{H}_{2} \mathrm{O}$ in the air to form $\mathrm{Li}$ hydroxide $(\mathrm{LiOH})$, $\mathrm{Li}$ nitride $(\mathrm{Li} 3 \mathrm{~N})$, and $\mathrm{Li}$ carbonate $\left(\mathrm{Li}_{2} \mathrm{CO}_{3}\right)$ [20], [21]. A day later, the Li metal turned completely white. When the Li metal turned black, no significant difference is observed from the surface of the initial Li metal in the SEM images, but after one day, numerous cracks are observed on the surface. Such contamination of the Li metal surface reduces the performance of the Li metal battery by causing the non-uniform current density when the battery is operated [22]. 

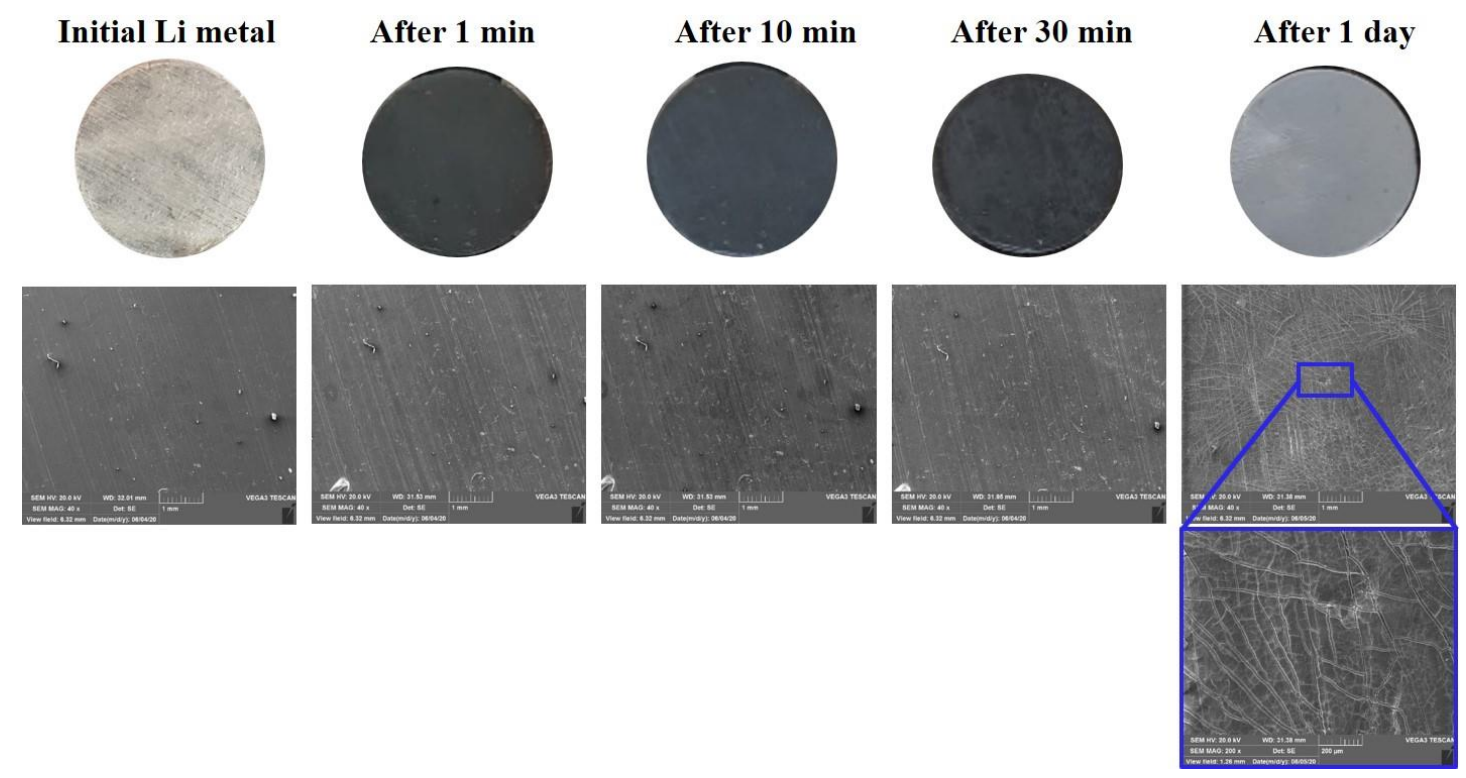

\section{Figure 1 Chemical change of Li metal in air}

In this paper, we discuss laser processing on Li metal using chamber, which has rarely been reported. Firstly, a chamber is designed and manufactured to maintain low RH. The chamber has three main parts: container lid with transparent plate glass, blocking frame, and specimen stage. The internal temperature, $\mathrm{RH}$, and dew point are measured to analyze the performance of the chamber, and the validation of the chamber is verified. In addition, when a Li metal is processed using a chamber, the laser beam interacts with the Li metal after passing the transparent glass plate. Therefore, the laser beam characteristics after passing the transparent glass plate are analyzed. Finally, laser ablation experiments of Li metal are carried out using the manufactured chamber to verify the system. 


\section{Concept of low relative humidity maintenance chamber for}

\section{laser processing}

\subsection{Design and manufacturing chamber}

(a) Schematic of chamber

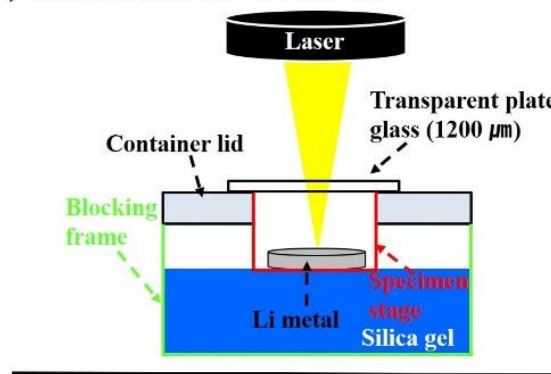

(c) Right side view

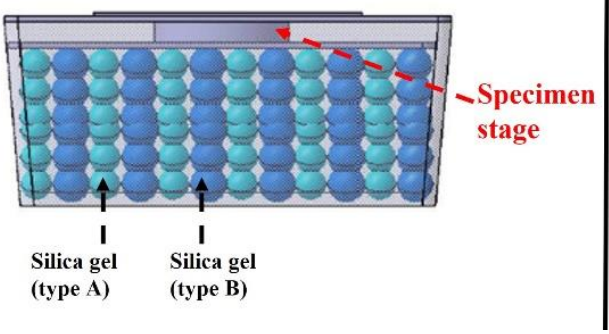

(b) Isometric view

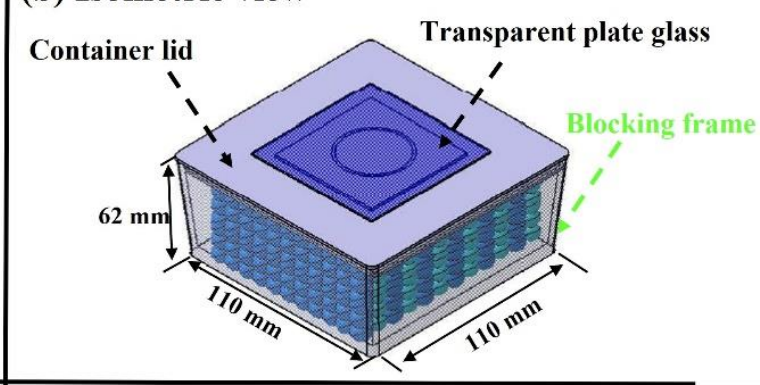

(d) Finished product

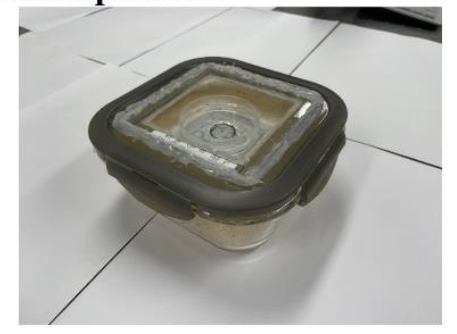

Figure 2 (a) Schematic of low relative humidity maintenance chamber; (b) Isometric view and (c) right side view of the laser processing chamber; (d) Image of finished product;

Table 1 The detailed information on silica gel

\begin{tabular}{|c|c|c|c|}
\hline \multirow{2}{*}{ Properties } & Type & A & B \\
\hline \multirow{3}{*}{ Adsorption } & RH $=\mathbf{2 0 \%}$ & 8.5 & 4.0 \\
\cline { 2 - 4 } & $\mathrm{RH}=\mathbf{5 0 \%}$ & $\mathbf{2 3 . 0}$ & $\mathbf{1 2 . 0}$ \\
\cline { 2 - 4 } & $\mathrm{RH}=\mathbf{9 0 \%}$ & 32.0 & $\mathbf{6 0 . 0}$ \\
\hline \multicolumn{2}{|c|}{ Diameter } & $2.5 \mathrm{~mm}$ & $3.5 \mathrm{~mm}$ \\
\hline \multicolumn{2}{|c|}{ Surface area } & $773.60 \mathrm{~m}^{2} / g$ & $574.30 \mathrm{~m}^{2} / g$ \\
\hline \multicolumn{2}{|c|}{ Pore diameter } & $22 \AA$ & $66 \AA$ \\
\hline
\end{tabular}

The boundary conditions required for chamber manufacturing are defined as follows. 
A. The dew point in the chamber should be below $-15{ }^{\circ} \mathrm{C}$

B. Fluid entering or leaving the chamber should be blocked.

C. Laser beam irradiated from outside should be transferable to Li metal inside the chamber.

To meet the above conditions, container lid, transparent plate glass, blocking frame, and silica gel are used in manufacturing the chamber. Figure 2 shows a schematic of chamber, 3D modeling, and finished products.

For the A condition, as mentioned above, Li metal is oxidized by rapidly reacting with oxygen and nitrogen in the air. In other words, maintaining the low RH in the air can slow down the reaction between oxygen and nitrogen as much as possible. Here, the criteria are defined as dew point because $\mathrm{RH}$ varies with temperature and pressure. The dew point means the amount of moisture in the air regardless of the temperature and pressure. Moreover, the reason for setting the minimum dew point of -15 is that Li metal can have been treated at $-15{ }^{\circ} \mathrm{C}$ in previous studies [23]. To keep the internal dew point of the chamber, silica gel is used. As shown in Figure 2, the position of the silica gel is located at the bottom of the specimen stage and inside the blocking frame. A-type and B-type of silica gel are utilized, and present physicochemical property of each silica gel in Table 1. A-type silica gel has a surface area of $773.60 \mathrm{~m}^{2} / \mathrm{g}$ and a pore diameter of $22 \AA$ and is effective in relatively low RH environments. On the other hand, B-type silica gel has a surface area of $574.30 \mathrm{~m}^{2} / \mathrm{g}$, and a pore diameter of $66 \AA$ and is effective at relatively high RH [24]. For this reason, two different silica gels are used. The two silica gels are mixed at a volume ratio of $1: 1$ to fill about $80 \%$ of the chamber volume.

In the case of condition $\mathrm{B}$, because the dew point is not kept stable when high $\mathrm{RH}$ air flows into the chamber, the container lid and blocking frame block the entering fluid from the outside.

In the $\mathrm{C}$ condition, transparent plate glass made of silicon dioxide $\left(\mathrm{SiO}_{2}\right)$ is used because laser beam externally irradiated must be reliably transferred to the surface of Li metal. Transparent plate glass 
is bound to container lid as shown in Figure 2.

\section{Characteristic analysis of low relative humidity maintenance chamber}

\subsection{Measurement of temperature, humidity, and dew point}

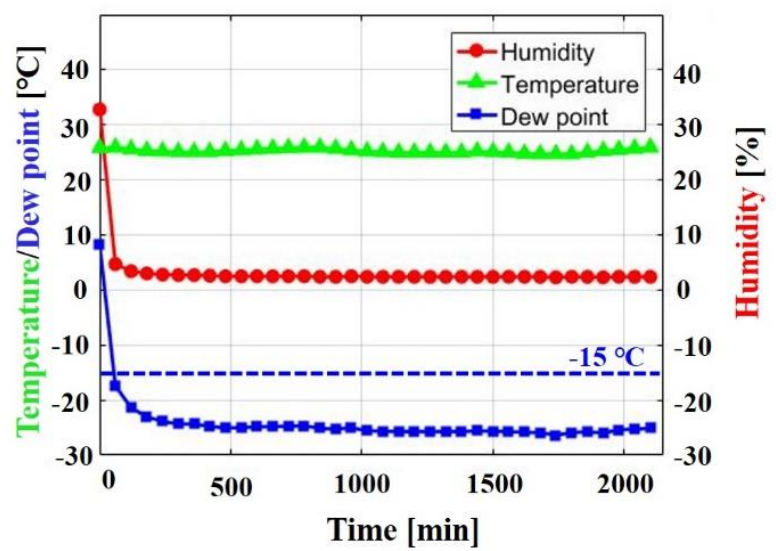

Figure 3 Change of temperature, relative humidity, and dew point inside chamber

$$
\text { Dew point }\left[{ }^{\circ} \mathrm{C}\right]=\frac{243.04\left[\ln \left(\frac{R H}{100}\right)+\frac{17.625 T}{243.04+T}\right]}{7.625-\ln \left(\frac{R H}{100}\right)-\frac{17.625 T}{243.04+T}}
$$

To evaluate the chamber performance, a thermo-hygrometer (Testo 623) is installed inside the chamber to measure the temperature (T) and RH. The dew point is calculated through Magnus formula, which is defined as Eq.1 [25]. Variation of temperature, $\mathrm{RH}$, dew point inside the chamber depending on time are shown in Figure 3. The initial conditions inside the chamber are temperature of $25.8^{\circ} \mathrm{C}, \mathrm{RH}$ of $32.8 \%$, and dew point of $8.2{ }^{\circ} \mathrm{C}$. According to the analysis, the temperature inside the chamber is maintained at approximately $24.7 \sim 25.9{ }^{\circ} \mathrm{C}$, and the $\mathrm{RH}$ decreases rapidly in the early stages, reaching 
$4.7 \%$ after 60 minutes. And then the RH keeps approximately $2.3 \sim 2.8 \%$ after 240 minutes. The dew point drop to $-17.4{ }^{\circ} \mathrm{C}$ in 60 minutes and continue to maintain $-23.8{ }^{\circ} \mathrm{C} \sim-26.4{ }^{\circ} \mathrm{C}$ after 240 minutes. Therefore, since the manufactured chamber can maintain a dew point of below $-15{ }^{\circ} \mathrm{C}$, it is implied that laser processing is possible by minimizing Li metal contamination caused by moisture.

\subsection{Analysis of gaussian beam characteristics}

We analyze the effect of transparent glass plate on Gaussian beam characteristics. Gaussian beam distribution can be represented as Eq.2 [26], [27].

$$
I(r)=I_{0} \exp \left(-\frac{2 r^{2}}{w_{0}^{2}}\right)=\frac{2 P}{\pi w_{0}^{2}} \exp \left(-\frac{2 r^{2}}{w_{0}^{2}}\right)
$$

where $I_{0}$ is peak intensity, $P$ is laser power, $r$ is the distance to the center of the beam, and $w_{0}$ is beam waist. Therefore, analysis of $P$, and $w_{0}$ is required to understand the Gaussian beam that changed after passing the transparent glass plate. 
(a)

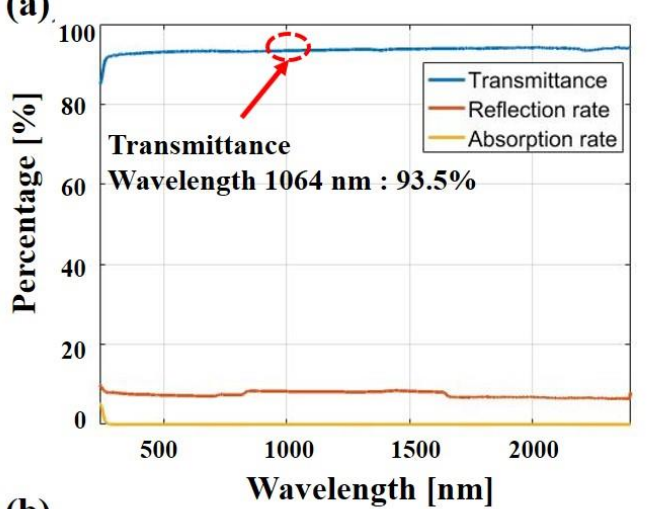

(b)

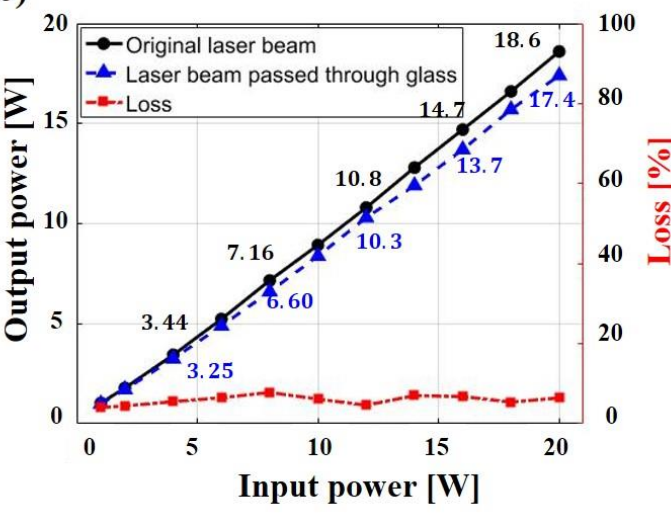

Laser

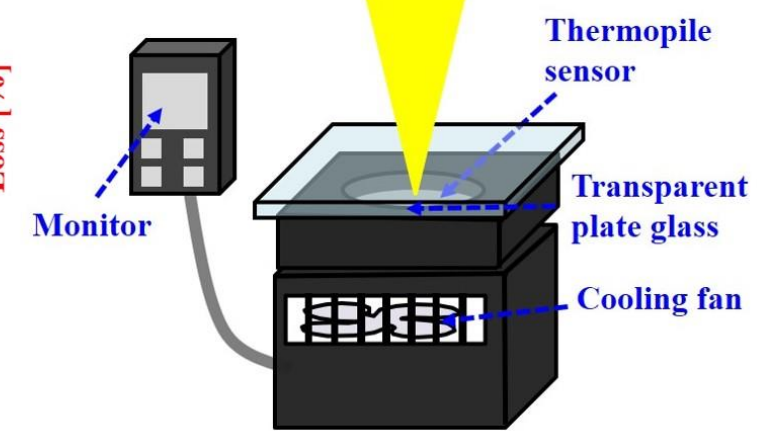

Figure 4 (a) Transmittance, reflection rate, and absorption rate of transparent plate glass; (b) Measurement of loss output power of beam passed through transparent plate glass;

Figure 4(a) is the result of measuring the transmittance, absorption, and reflectance of glass used in the chamber using a spectrophotometer (solidspec-3700). In wavelengths of $200 \mathrm{~nm}$ to 2400 $n m$, the measured transmittance is $85.0 \% \sim 94.1 \%$.

The power loss is analyzed when the Gaussian beam passes through the glass using a nanosecond ytterbium fiber laser with a wavelength of $1064 \mathrm{~nm}$ (YLPM-1-4x200-20-20) and laser power meters (PM300F-19, Coherent). The laser processing parameters for the experiment are fixed with a pulse duration of $4 \mathrm{~ns}$ and a pulse repetition rate of $500 \mathrm{kHz}$, and then the laser power is changed from $1 \mathrm{~W}$ to $20 \mathrm{~W}$. Moreover, when calculating the rate of power loss, the power loss inside the optical system is not included. As displayed in Figure 4 (b), the rate of power loss of Gaussian beam passing through glass is $4.06 \% \sim 7.03 \%$. The rate of power loss is in good agreement with the result of the absorption rate and reflectance rate of glass, although there are slight errors depending on the input 
power.

(a)

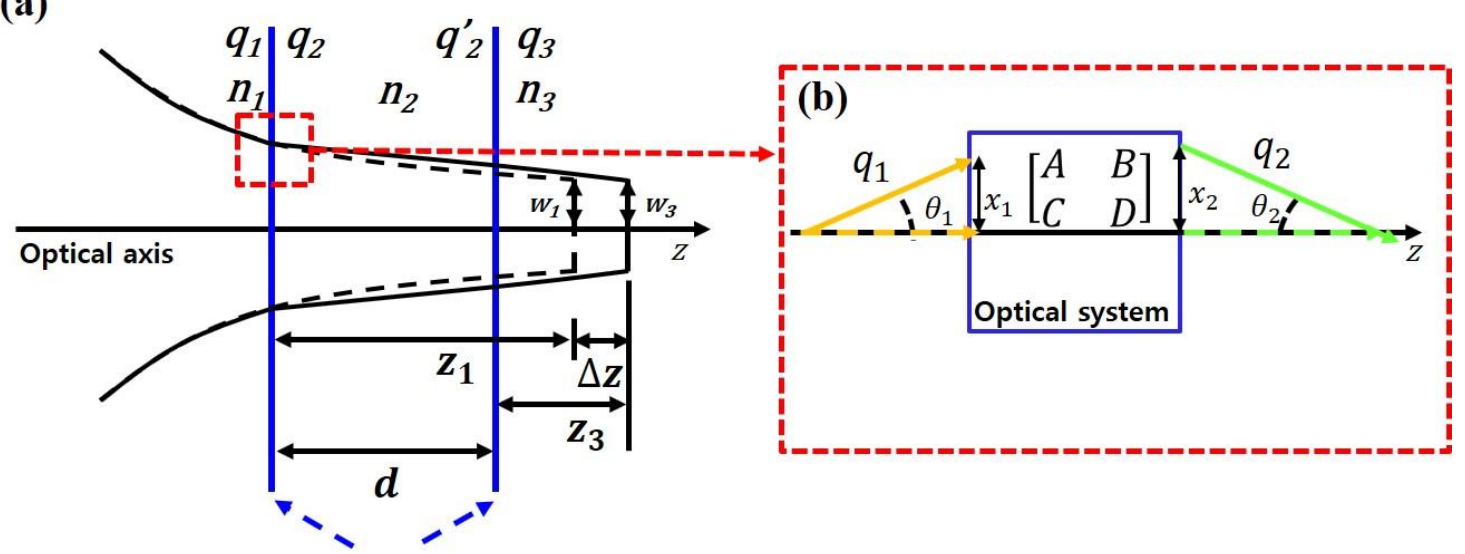

Interface

Figure 5 Variation of beam waist and focal length when gaussian beam passes through two interfaces.

$$
\begin{gathered}
\frac{1}{q(z)}=\frac{1}{R(z)}-\frac{1}{n k w(z)^{2}} j \\
w(z)=w_{0}\left[1+\left(\frac{z}{n k w_{0}^{2}}\right)^{2}\right]^{\frac{1}{2}} \\
R(z)=z\left[1+\left(\frac{n k w_{0}^{2}}{z}\right)^{2}\right]
\end{gathered}
$$

In Figure 5 (a), the Gaussian beam propagating along the z-axis (optical axis) on a uniform medium can be represented Eq.3 [28]-[30]. Where, $q$ is complex beam parameter, $R$ is radius of the phase front of the beam, $w$ is beam waist, $k\left(\frac{2 \pi}{\lambda}\right)$ is wavenumber, $\lambda$ is wavelength , and $n$ is refractive index. From Eq.3 - 5, Eq. 6 can be obtained.

$$
q_{i}(z)=z_{i}+j n_{i} k w_{i}^{2}(i=1,2,3,4 \ldots)
$$


When the Gaussian beam passes through the optical system, the relationship between the beam before passing $\left(q_{1}\right)$ and the beam after passing $\left(q_{2}\right)$ can be obtained through $\mathrm{ABCD}$ matrix. ABCD matrix is $2 \times 2$ matrix that allows a beam to be tracked according to elements of the optical system when a beam has paraxial ray properties, such as a Gaussian beam. Elements A, B, C and D of Matrix are determined by the optical system, and the relational expression can write it down as shown in Eq.7 [30].

$$
q_{2}=\left(\begin{array}{ll}
A & B \\
C & D
\end{array}\right) q_{1}
$$

In Figure 5(b), $q_{i}$ can be represented by a column vector of height $x_{i}$, angle $\theta_{i}$ from the optical axis. So, we can rewrite it as Eq.8.

$$
\left(\begin{array}{l}
x_{2} \\
\theta_{2}
\end{array}\right)=\left(\begin{array}{ll}
A & B \\
C & D
\end{array}\right)\left(\begin{array}{l}
x_{1} \\
\theta_{1}
\end{array}\right)
$$

The matrix above is developed as follows:

$$
\begin{aligned}
& x_{2}=A x_{1}+B \theta_{1} \\
& \theta_{2}=C x_{1}+D \theta_{1}
\end{aligned}
$$

Here, the following conditions are established due to the characteristics of the paraxial ray.

$$
\sin \theta \approx \theta, \tan \theta \approx \theta, \cos \theta \approx 1
$$


By using Eq.11, Eq.9 and Eq.10 can be rewritten as follow:

$$
\begin{aligned}
& \frac{x_{2}}{\theta_{1}}=\frac{A x_{1}}{\theta_{1}}+B=A q_{1}+B \\
& \frac{\theta_{2}}{\theta_{1}}=\frac{C x_{1}}{\theta_{1}}+D=C q_{1}+D
\end{aligned}
$$

Next, divide Eq.12 by Eq.13 to get the following expression.

$$
q_{2}=\frac{A q_{1}+B}{C q_{1}+D}
$$

We can estimate the Gaussian beam change by Eq.14, when the Gaussian beam passes through a medium with a refractive index of $n_{1}$ to $n_{2}$.

Let’s estimate the change in Gaussian beam passing through two interfaces, such as glass.

$$
q_{1}(z)=z_{1}+j n_{1} k w_{1}^{2}, \quad q_{2}(z)=z_{2}+j n_{2} k w_{2}^{2}, \quad q_{3}(z)=z_{3}+j n_{3} k w_{3}^{2}
$$

When the Gaussian beam proceeds to a flat dielectric interface such as Figure 5(a), each element of ABCD matrix is $A=1, B=C=0, D=\frac{n_{1}}{n_{2}}[29]$. 


$$
q_{2}=\frac{1 q_{1}+0}{0 q_{1}+\frac{n_{1}}{n_{2}}}=\frac{n_{2}}{n_{1}} q_{1}
$$

From Eq.15 and Eq.16, we can obtain Eq.17. At this time, $w_{1}$ and $w_{2}$ are the same because $w_{1}$ and $w_{2}$ have the same wavelength of laser source, focal length of lens, and input beam diameter. [31].

$$
z_{2}=\frac{n_{2}}{n_{1}} z_{1}, \quad\left(w_{1}=w_{2}\right)
$$

If the Gaussian beam moves by $d$ on the same medium, each element of $\mathrm{ABCD}$ matrix is $A=1, B=$ $d, C=0, D=1$.

$$
q_{2}^{\prime}=\frac{1 q_{2}+d}{0 q_{2}+1}=q_{2}+d
$$

Next, if the Gaussian beam progresses from $n_{2}$ to $n_{3}$, it can be expressed in the same way as Eq.16.

$$
q_{3}=\frac{1 q_{2}^{\prime}+0}{0 q_{2}^{\prime}+\frac{n_{2}}{n_{3}}}=\frac{n_{3}}{n_{2}} q_{2}^{\prime}
$$

Eq. 20 can be obtained from Eq. 15 - 19. In this case, negative values must be substituted because the slope of the incident beam is less than zero. 


$$
z_{3}=\frac{n_{3}}{n_{1}} z_{1}-\frac{n_{3}}{n_{2}} d, \quad\left(w_{2}=w_{3}\right)
$$

$\Delta z$ can be calculated as follows:

$$
\Delta z=z_{3}+d-z_{1}=\left(1-\frac{n_{3}}{n_{2}}\right) d-\left(1-\frac{n_{3}}{n_{1}}\right) z_{1}
$$

For this study, since $n_{1}=n_{3}$, it can be simply calculated as follows:

$$
\Delta z=\left(1-\frac{n_{3}}{n_{2}}\right) d
$$

Eq. 20 indicates that if the Gaussian beam passes through transparent plate glass, the focal length is determined by the refractive index and thickness of the transparent plate glass. Therefore, the glass used in the chamber is approximately $1200 \mu \mathrm{m}$ of thickness and the refractive index is 1.42 , so that it is located approximately $353 \mu \mathrm{m}$ away from the original focal length.

\section{Experiments and results}

Section 4 conducts experiments to verify the theoretical approach in Section 3.2. Nanosecond pulsed laser (YLPM-1-4x200-20-20) is used a as laser processing source, and SM490A as a specimen is prepared for experiments. The laser processing parameters are power of $20 \mathrm{~W}$, wavelength of 1064 $\mathrm{nm}$, pulse duration of $8 \mathrm{~ns}$, pulse repletion rate of $200 \mathrm{kHz}$ and 40000 shots. Under the same parameters, the laser is irradiated by changing the distance from the specimen surface to the F-theta lens from $-1900 \mu m$ to $1900 \mu m$ (100 $\mu m$ interval). When the smallest and high roundness crater is 
formed after laser irradiation, that distance is defined as the focal length.

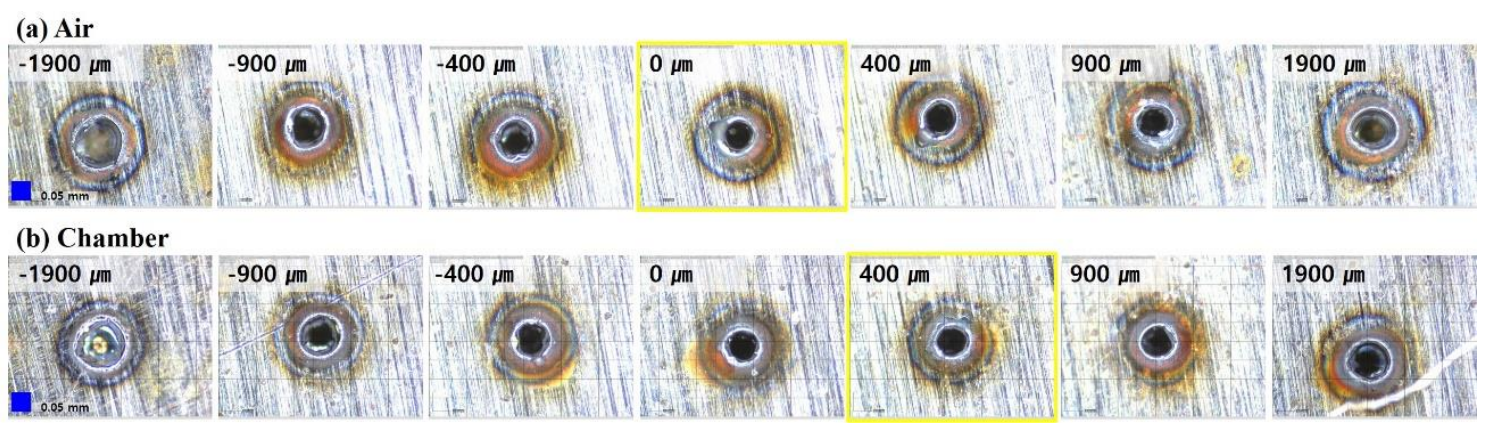

Figure 6 Optical microscope images of craters produced in SM490A with different focal length when experiments are carried out in (a) air and (b) chamber

Figure 6 displays an optical microscope image of craters formed in the SM490A after the experiments. Basically, the beam waist of the Gaussian beam decreases from the laser lens to the focal point. After that, the beam waist increases again. Therefore, both craters formed in the air and chamber is produced relatively wide craters at $-1900 \mu m$ and relatively small craters are produced near the focal point. At $1900 \mu m$, it can be observed that craters are formed relatively wider than near the focal point. 

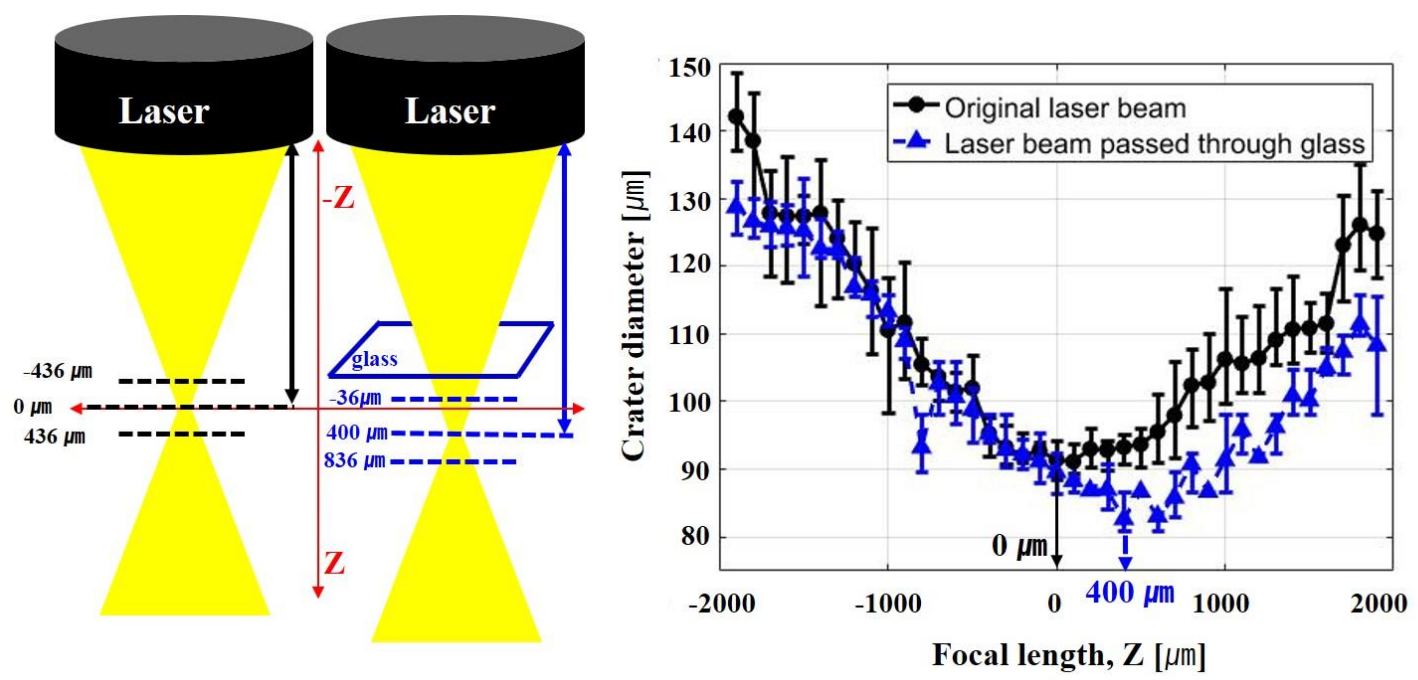

Figure 7 Comparison of focal length in original laser beam and laser beam passed through glass

Figure 7 shows crater diameters according to focal length. Like the previous optical microscope image, the diameter of the generated crater is smallest at the focal point. When comparing focal length of laser beam passed through plate glass to the original beam, the focal length is approximately 400 $\mu m$ away. This result has $13.31 \%$ error compared to the theoretically calculated value $(353 \mu m)$. In addition, each graph shows a relatively constant crater diameter near the focal length. This region means the depth of focus (DOF). The DOF of original laser beam is approximately $-436 \mu \mathrm{m} \sim 436 \mu \mathrm{m}$, and the DOF of laser beam passed through the glass moves as much as the focus has far away. Moreover, a crater diameter formed by the laser beam passed through glass is produced smaller than that of the original beam. This is because the power loss is caused by the glass. Hereby, the laser beam change after passing through the glass is experimentally verified. 


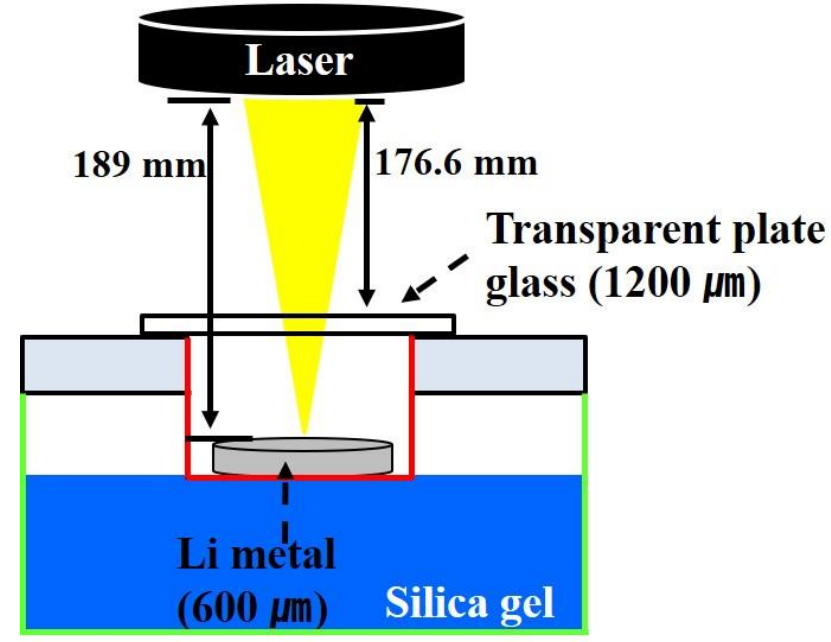

Figure 8 Schematic of validation experiments

Figure 8 shows a schematic of experiments to verify the possibility of Li metal laser processing using the manufactured chamber. The experiments are conducted with the same laser as above, and the Li metal (MTI Corporation, USA) is $600 \mu m$ thick. The laser parameters are pulse duration of $4 n s$, pulse repetition rate of $500 \mathrm{kHz}, 450$ shots, and pulse energy is applied at $1 \mu \mathrm{J}$ interval from $13 \mu \mathrm{J}$ to $22 \mu \mathrm{J}$.
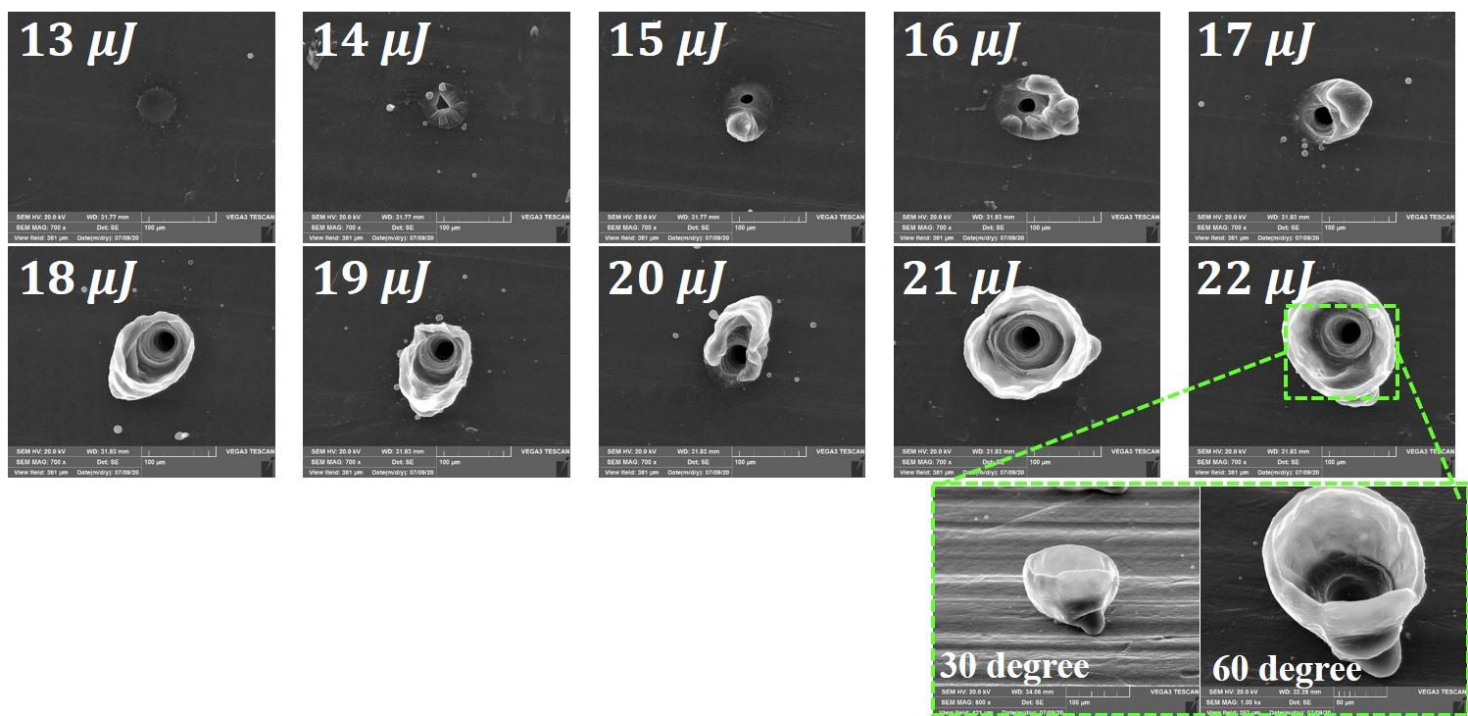

Figure 9 SEM images of craters produced in Li metal at $4 \mathrm{~ns}$ pulse duration, $500 \mathrm{kHz}$ pulse repetition rate, and 450 shots 
Figure 9 is an SEM image of crater formed after laser irradiation on Li metal. When looking at craters formed, traces of Li metal melting, evaporation, and melt expansion are observed. Because thermal ablation is dominant in nanosecond laser [32]. In addition, the crater diameter increases with increasing pulse energy. When pulse energy above $15 \mu \mathrm{J}$ is applied, burr begins to form. In particular, no contamination was observed on the surface of Li metal by moisture, and it was confirmed that $\mathrm{Li}$ metal laser processing was possible through manufactured chamber.

\section{Conclusion}

In this study, a low humidity maintenance chamber was manufactured to apply the laser process to Li metal. The performance of the manufactured chamber was evaluated and the effect of plate glass on Gaussian beam was analyzed. Finally, the possibility of laser processing of Li metal was confirmed through experiments. The main results of this study are as follows:

1. The dew point inside the manufactured chamber fell to $-17.4{ }^{\circ} \mathrm{C}$ in 60 minutes. After 240 minutes, the dew point was maintained at $-23.8{ }^{\circ} \mathrm{C} \sim-26.4{ }^{\circ} \mathrm{C}$. As a result, contamination of $\mathrm{Li}$ metal by $\mathrm{H}_{2} \mathrm{O}$ could be prevented through the manufactured chamber.

2. When the Gaussian beam passed through the plate glass, the laser power was lost due to absorption and reflection of the glass, and the focal length was determined depending on the thickness and reflective index of the plate glass.

3. Laser ablation of Li metal was conducted using a chamber. Like other metal laser ablation, traces of melting, evaporation, and melt expulsion were observed. In addition, no contamination of Li metal was observed after experiments. Therefore, it is believed that $\mathrm{Li}$ metal laser processing is possible through the manufactured chamber. 
Future plans will study the interaction characteristics between lithium metal and nanosecond laser using the manufactured chamber. In particular, laser ablation thresholds and incubation coefficients are studied.

\section{Acknowledgment}

The research described herein was sponsored by the National Research Foundation of Korea (NRF) grant funded by the Korean government (MSIP; Ministry of Science, ICT \& Future Planning) (No. 2019R1A2C1089644) and by the Korea Innovation Foundation grant funded by the Korean Government (MSIP) (No. 2020-DD-SB-0159).

\section{Reference}

[1] G. Zubi, R. Dufo-López, M. Carvalho, and G. Pasaoglu, "The lithium-ion battery: State of the art and future perspectives,” Renew. Sustain. Energy Rev., vol. 89, no. October 2017, pp. 292-308, 2018.

[2] N. Nitta, F. Wu, J. T. Lee, and G. Yushin, "Li-ion battery materials: Present and future," Mater. Today, vol. 18 , no. 5 , pp. 252-264, 2015.

[3] C. M. Julien and A. Mauger, "NCA, NCM811, and the route to Ni-Richer lithium-Ion batteries," Energies, vol. 13, no. 23, 2020.

[4] J.-H. Rakebrandt, P. Smyrek, Y. Zheng, H. J. Seifert, and W. Pfleging, “ Laser processing of thick Li(NiMnCo)O 2 electrodes for lithium-ion batteries ," Laser-based Micro- Nanoprocessing XI, vol. 10092, p. 100920M, 2017.

[5] J. Sourice et al., "Core-shell amorphous silicon-carbon nanoparticles for high performance anodes in lithium ion batteries," J. Power Sources, vol. 328, pp. 527-535, 2016. 
[6] J. Qian et al., "High rate and stable cycling of lithium metal anode," Nat. Commun., vol. 6, 2015.

[7] J. Lang, L. Qi, Y. Luo, and H. Wu, "High performance lithium metal anode: Progress and prospects," Energy Storage Mater., vol. 7, no. December 2016, pp. 115-129, 2017.

[8] R. Wang, W. Cui, F. Chu, and F. Wu, "Lithium metal anodes: Present and future," J. Energy Chem., vol. 48, pp. 145-159, 2020.

[9] Z. Luo et al., "Interfacial challenges towards stable Li metal anode," Nano Energy, vol. 79, no. August 2020, p. 105507, 2021.

[10] Q. Li, S. Zhu, and Y. Lu, "3D Porous Cu Current Collector/Li-Metal Composite Anode for Stable Lithium-Metal Batteries," Adv. Funct. Mater., vol. 27, no. 18, 2017.

[11] J. Yang, C. Y. Wang, C. C. Wang, K. H. Chen, C. Y. Mou, and H. L. Wu, “Advanced nanoporous separators for stable lithium metal electrodeposition at ultra-high current densities in liquid electrolytes," J. Mater. Chem. A, vol. 8, no. 10, pp. 5095-5104, 2020.

[12] J. Park, J. Jeong, Y. Lee, M. Oh, M. H. Ryou, and Y. M. Lee, "Micro-Patterned Lithium Metal Anodes with Suppressed Dendrite Formation for Post Lithium-Ion Batteries,” Adv. Mater. Interfaces, vol. 3, no. 11 , pp. 1-9, 2016.

[13] T. Jansen, D. Blass, S. Hartwig, and K. Dilger, "Processing of Advanced Battery Materials-Laser Cutting of Pure Lithium Metal Foils," Batteries, vol. 4, no. 3. p. 37, 2018.

[14] S. Chatterjee, S. S. Mahapatra, V. Bharadwaj, B. N. Upadhyay, and K. S. Bindra, "Prediction of quality characteristics of laser drilled holes using artificial intelligence techniques," Eng. Comput., vol. 37, no. 2, pp. 1181-1204, 2021.

[15] M. C. Rossi, J. M. Amado, M. J. Tobar, A. Vicente, A. Yañez, and V. Amigó, "Effect of alloying elements on laser surface modification of powder metallurgy to improve surface mechanical properties of beta titanium alloys for biomedical application," J. Mater. Res. Technol., vol. 14, pp. 1222-1234, 2021.

[16] C. Chen, X. Yin, W. Liao, Y. Xiang, M. Gao, and Y. Zhang, "Microstructure and properties of 6061/2A12 dissimilar aluminum alloy weld by laser oscillation scanning," J. Mater. Res. Technol., vol. 
14, pp. 2789-2798, 2021.

[17] D. Lee and J. Suk, "Laser cutting characteristics on uncompressed anode for lithium-ion batteries," Energies, vol. 13, no. 10, 2020.

[18] S. Son and D. Lee, "The effect of laser parameters on cutting metallic materials," Materials (Basel)., vol. 13, no. 20, pp. 1-15, 2020.

[19] L. N. Trinh and D. Lee, "The characteristics of laser welding of a thin aluminum tab and steel battery case for lithium-ion battery," Metals (Basel)., vol. 10, no. 6, pp. 1-15, 2020.

[20] X. Shen et al., "Lithium anode stable in air for low-cost fabrication of a dendrite-free lithium battery," Nat. Commun., vol. 10, no. 1, 2019.

[21] K. Liao et al., "Developing a 'Water-Defendable' and 'Dendrite-Free' Lithium-Metal Anode Using a Simple and Promising GeCl4 Pretreatment Method," Adv. Mater., vol. 30, no. 36, pp. 1-8, 2018.

[22] D. Aurbach et al., "Recent studies of the lithium-liquid electrolyte interface Electrochemical, morphological and spectral studies of a few important systems," J. Power Sources, vol. 54, no. 1, pp. 76-84, 1995.

[23] M. H. Cho et al., "The effects of moisture contamination in the Li-O2 battery," J. Power Sources, vol. 268, pp. 565-574, 2014.

[24] X. Li, Z. Li, Q. Xia, and H. Xi, "Effects of pore sizes of porous silica gels on desorption activation energy of water vapour," Appl. Therm. Eng., vol. 27, no. 5-6, pp. 869-876, 2007.

[25] M. G. Lawrence, "The relationship between relative humidity and the dewpoint temperature in moist air: A simple conversion and applications,” Bull. Am. Meteorol. Soc., vol. 86, no. 2, pp. 225-233, 2005.

[26] F. Di Niso, C. Gaudiuso, T. Sibillano, F. P. Mezzapesa, A. Ancona, and P. M. Lugarà, "Role of heat accumulation on the incubation effect in multi-shot laser ablation of stainless steel at high repetition rates," Opt. Express, vol. 22, no. 10, p. 12200, 2014.

[27] F. Li, X. Chen, W. Lin, H. Pan, X. Jin, and X. Hua, "Nanosecond laser ablation of Al-Si coating on boron steel," Surf. Coatings Technol., vol. 319, pp. 129-135, 2017.

[28] S. Nemoto, "Waist shift of a Gaussian beam by a dielectric plate," Appl. Opt., vol. 28, no. 9, pp. 1643- 
$1647,1989$.

[29] S. Nemoto, "Waist shift of a Gaussian beam by a dielectric interface," Appl. Opt., vol. 27, no. 9, pp. $1833-1839,1988$.

[30] J. Alda, "Laser and Gaussian Beam Propagation and Transformation," Encycl. Opt. Eng., pp. 999-1013, 2003.

[31] S. Jeong and S. H. Lee, "Effects of Process Parameters on Laser Ablation Based Machining and Measurements," J. Korean Soc. Precis. Eng., vol. 28, no. 12, pp. 1359-1365, 2011.

[32] K. H. Leitz, B. Redlingshöer, Y. Reg, A. Otto, and M. Schmidt, "Metal ablation with short and ultrashort laser pulses," Phys. Procedia, vol. 12, no. PART 2, pp. 230-238, 2011. 